\begin{tabular}{|c|l|}
\hline Title & Selective mono-fluorination of diols via a cyclic acetal of N,N-diethyl-4-methoxybenzamide \\
\hline Author(s) & Suwada, Mitsuhiro; Fukuhara, Tsuyoshi; Hara, Shoji \\
\hline Citation & $\begin{array}{l}\text { Journal of Fluorine Chemistry, 128(10), 1121-1125 } \\
\text { https://doi.org/10.1016/.jluchem.2007.04.020 }\end{array}$ \\
\hline Issue Date & $2007-10$ \\
\hline Doc URL & http://hdl.handle.net/2115/30297 \\
\hline Type & article (author version) \\
\hline File Information & JFC128 10.pdf \\
\hline
\end{tabular}

Instructions for use 


\title{
Selective mono-fluorination of diols via a cyclic acetal of $N, N$-diethyl-4-methoxybenzamide
}

\author{
Mitsuhiro Suwada, Tsuyoshi Fukuhara, Shoji Hara* \\ Division of Chemical Process Engineering, Graduate School of Engineering, \\ Hokkaido University, Sapporo 060-8628, Japan
}

\begin{abstract}
Selective mono-fluorination of 1,2- and 1,3-diols was achieved using $N, N$-diethyl-4-methoxybenzamide diethyl acetal and $\mathrm{Et}_{3} \mathrm{~N}-3 \mathrm{HF}$. The reaction proceeds through a cyclic acetal of the benzamide, and only one hydroxy group was fluorinated and another one was acylated.

Keywords: Mono-fluorination of diol; Benzamide acetal; Triethylamine trishydrogen fluoride
\end{abstract}

\section{Introduction}

Recently, we succeeded in the selective mono-fluorination of 1,2- and 1,3-diols using $N, N$-diethyl- $\alpha, \alpha$-difluoro-4-methylbenzylamine (DFMBA), and a cyclic acetal of the benzamide was expected to be formed as an intermediate from DFMBA and the diol [1]. In this paper, we prepared the cyclic amide acetals from amide diethyl acetals and diols, and converted them to the mono-fluorination products by $\mathrm{Et}_{3} \mathrm{~N}-3 \mathrm{HF}$.

\section{Result and discussion}

The cyclic acetal of $N, N$-diethyl-3-methylbenzamide (1a), which is an expected

${ }^{*}$ Corresponding author. Fax: +81-11-706-6556

e-mail address: hara@org-mc.eng.hokudai.ac.jp 
intermediate in the reaction of ethylene glycol with DFMBA [1], was prepared by transacetalization from $N, N$-diethyl-3-methylbenzamide diethyl acetal and ethylene glycol [2], and was applied to the fluorination without isolation. When the reaction of 1a with $\mathrm{Et}_{3} \mathrm{~N}-3 \mathrm{HF}$ was carried out at $140{ }^{\circ} \mathrm{C}$ for $30 \mathrm{~min}$, 2-fluoroethyl 3-methylbenzoate (2a), which is the same product as in the reaction of ethylene glycol with DFMBA [1], was obtained in $66 \%$ yield. Similarly, the cyclic acetal of $N, N$-diethylbenzamide (1b), $N, N$-diethyl-4-chlorobenzamide (1c), and $N, N$-diethyl-4-methoxybenzamide (1d) were prepared from the corresponding bezamide diethyl acetal and ethylene glycol, and used for the fluorination reaction under the same conditions. Among them, 1d gave the best result and 2-fluoroethyl 4-methoxybenzoate (2d) was obtained in 80\% yield (Table 1). From the cyclic acetals of DMF and DMA, the corresponding fluorination products could not be obtained under the same conditions.

Table 1 Fluorination of ethylene glycol using benzamide diethyl acetals ${ }^{a}$

\begin{tabular}{lll}
\hline $\mathbf{1}(\mathrm{X})$ & Product $\mathbf{2}(\mathrm{X})$ & Yield (\%) \\
\hline $\mathbf{1 a}(3-\mathrm{Me})$ & $\mathbf{2 a}(3-\mathrm{Me})$ & 66 \\
$\mathbf{1 b}(\mathrm{H})$ & $\mathbf{2 b}(\mathrm{H})$ & 54 \\
$\mathbf{1 c}(4-\mathrm{Cl})$ & $\mathbf{2 c}(4-\mathrm{Cl})$ & 57 \\
$\mathbf{1 d}(4-\mathrm{MeO})$ & $\mathbf{2 d}(4-\mathrm{MeO})$ & 80 \\
\hline
\end{tabular}

a Transacetalization was carried out using 1.1 eq of acetal under reduced pressure at $50^{\circ} \mathrm{C}$ for $1 \mathrm{~h}$. Fluorination was carried out at $140^{\circ} \mathrm{C}$ for $30 \mathrm{~min}$ using 2 eq of $\mathrm{Et}_{3} \mathrm{~N}-3 \mathrm{HF}$.

${ }^{\mathrm{b}}$ Isolated yield based on ethylene glycohol .

The cyclic acetal intermediate $\mathbf{1 d}$ is isolable by distillation after the transacetalization, and from the isolated $\mathbf{1 d}$, $2 \mathbf{d}$ could be obtained in $83 \%$ yield by the reaction with $\mathrm{Et}_{3} \mathrm{~N}-3 \mathrm{HF}$ at $140{ }^{\circ} \mathrm{C}$ for $30 \mathrm{~min}$. These results showed that the reaction 
proceeds through the cyclic acetal intermediate $\mathbf{1}$ as expected. The reaction from $\mathbf{1}$ to $\mathbf{2}$ must be proceeding as follows: Elimination of diethylamine from $\mathbf{1}$ took place to give a dioxolenium intermediate [3], and subsequent fluoride attack, generated from DFMBA or $\mathrm{Et}_{3} \mathrm{~N}-3 \mathrm{HF}$, on the dioxolenium intermediate gave the fluoroethyl benzoate 2 (Scheme 1).

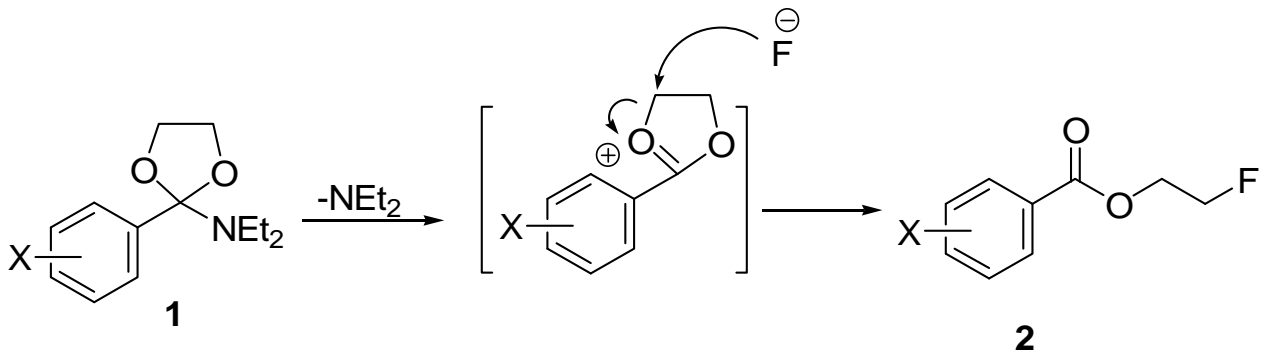

Scheme 1

In order to optimize the reaction conditions, the fluorination reaction of $\mathbf{1 d}$ was carried out under various conditions. Lower temperature $\left(120^{\circ} \mathrm{C}\right)$ or shorter reaction time (10 min) caused the decrease of the yield. On the other hand, higher temperature $\left(160{ }^{\circ} \mathrm{C}\right)$ or longer reaction time $(1 \mathrm{~h})$ could not improve the result. We also applied various fluoride sources such as $\mathrm{KF}$, TBAF, $\mathrm{Et}_{3} \mathrm{~N}-2 \mathrm{HF}$, and $\mathrm{Et}_{3} \mathrm{~N}-4 \mathrm{HF}$, but the results could not be improved. The present method was applied to the reaction with various 1,2- and 1,3-diols (Table 2). From cis-1,2-cyclohexanediol, trans-2-fluorocyclohexyl 4-methoxybenzoate (3) was obtained in 75\% yield (Entry 1). The reaction proceeded with inversion of the stereochemistry. On the other hand, from trans-1,2-cyclohexanediol, trans-2-fluorocyclohexanol was obtained in low yield and the expected methoxybenzoyl ester of cis-2-fluorocyclohexanol was not formed. In this case, the formation of the cyclic acetal is slow due to the steric effect and a cyclohexene oxide must be initially formed by the reaction of the trans-cyclohexanediol with amide acetal [4]. The subsequent ring opening fluorination of the epoxide by $\mathrm{Et}_{3} \mathrm{~N}-3 \mathrm{HF}$ provided trans-2-fluorocyclohexanol [5]. In the reaction with 1,2-dodecanediol, the fluorination took place non-regioselectively to give a mixture of 1- and 2-fluorinated products (4a : $\mathbf{4 b}=71: 29$ ) (Entry 2). In the reaction of cis-1,2-cyclododecandiol, the resulting trans-2-fluorocyclododecyl 4-methoxybenzoate was subjected to the transesterification reaction without isolation [6] and 
trans-2-fluorocyclododecanol (5) was obtained in 56\% overall yield from cyclododecandiol (Entry 3). As the reaction proceeds stereoselectively, the optically active fluorohydrin derivatives could be selectively prepared from optically active diols without losing their original enantiomeric purity. For instance, when $(R, R)$-hydrobenzoin was subjected to the reaction, $(1 R, 2 S)$-2-fluoro-1,2-diphenylethyl 4-methoxybenzoate (7) was obtained in 77\% yield (>95\%de) (Entry 5). Its absolute stereochemistry was determined by comparison of its optical rotation with the product from DFMBA after converting to the 3-methylbenzoic acid ester (Scheme 2). Similarly, from $(R, R)$-2,3-butanediol, (2R, 3S)-3-fluoro-2-butyl 4-methoxybenzoate (6) was obtained in $79 \%$ yield (>95\%de) (Entry 4 ).

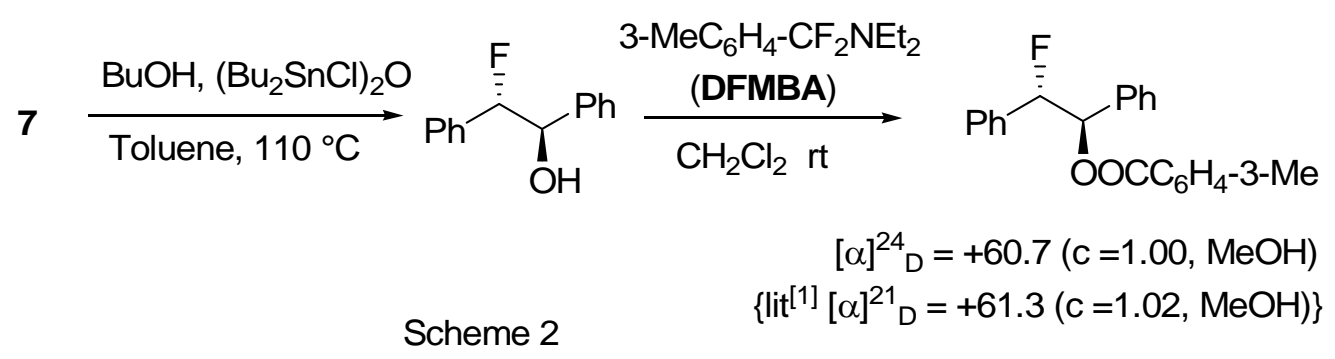

The reaction of 1,3-diols is sluggish and higher temperature $\left(160{ }^{\circ} \mathrm{C}\right)$ was required to complete the reaction (Entries 6 and 7). Recently, we found that the reaction of $\beta$-amino alcohols with DFMBA also proceeds through the similar cyclic intermediate to give fluoroalkylamides [7]. The reaction of $N$-phenyl-2-aminoethanol and $\mathrm{N}$-phenyl-1-amino-2-octanol with the benzamide diethyl acetal and $\mathrm{Et}_{3} \mathrm{~N}-3 \mathrm{HF}$ proceeded as in the case of DFMBA and $N$-acylated 2-fluoroethylamine (10) and 2-fluorooctylamine (11) were obtained, respectively (Entries 8 and 9). 
Table 2 Fluorination of diols and amino alcohol using p-methoxybenzamide diethyl acetal and $\mathrm{Et}_{3} \mathrm{~N}-3 \mathrm{HF}^{\mathrm{a}}$

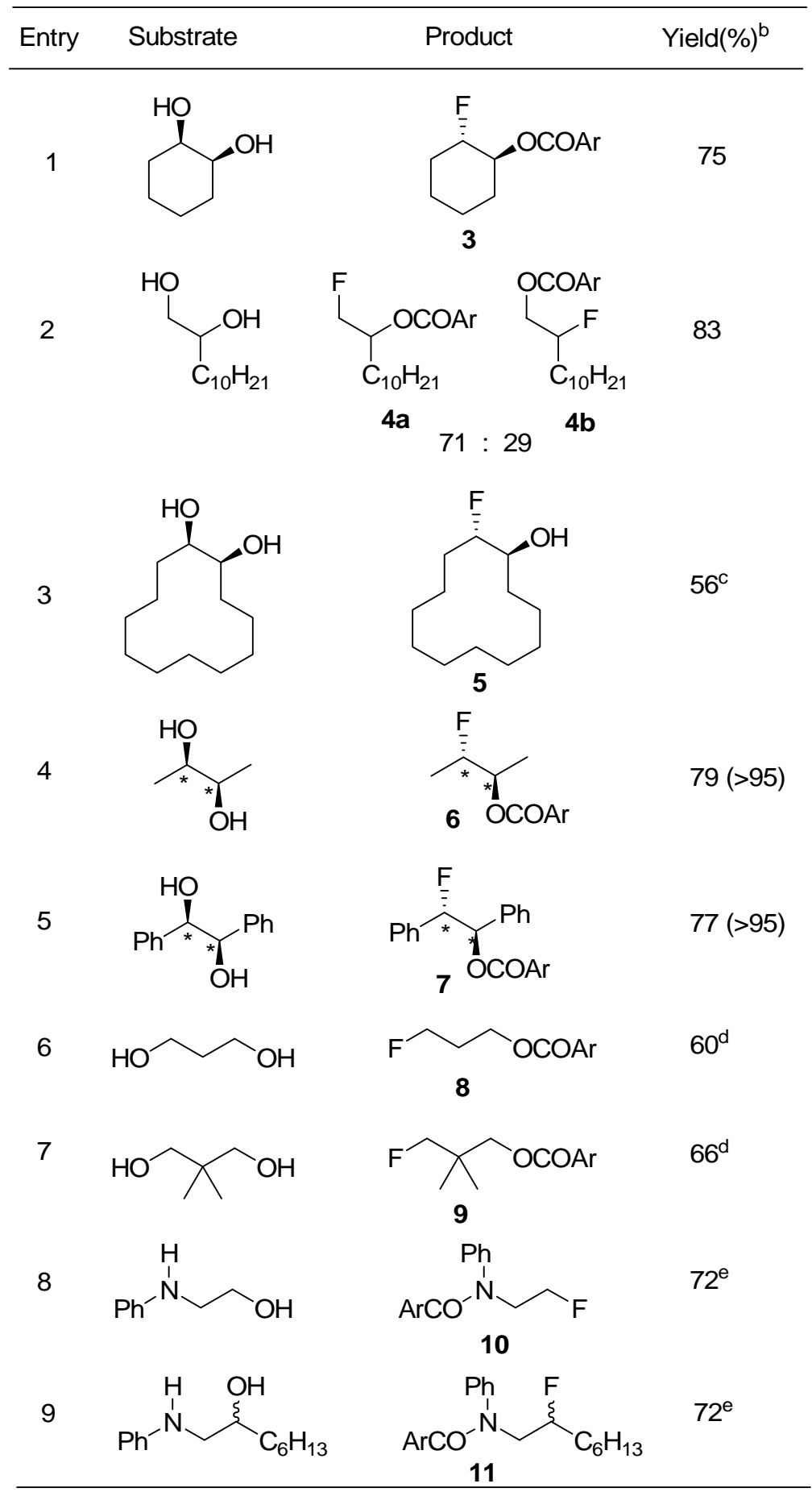

alf otherwise not mentioned, the reaction was carried out at $140{ }^{\circ} \mathrm{C}$ for $30 \mathrm{~min}$. $\mathrm{Ar}=p-\mathrm{MeOC}_{6} \mathrm{H}_{4}$.

${ }^{b}$ Isolated yield based on diol or amino alcohol used and in paretheses, diasteromeric excess.

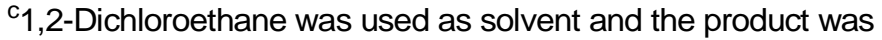
converted to fluorohydrin before isolation.

${ }^{\mathrm{d}}$ The reaction was carried out at $160{ }^{\circ} \mathrm{C}$ for $30 \mathrm{~min}$. ${ }^{\mathrm{e}}$ The reaction was carried out at $100{ }^{\circ} \mathrm{C}$ for $30 \mathrm{~min}$. 


\section{Experimental}

\subsection{General methods}

The melting points were measured with a Yanagimoto micro melting-point apparatus and uncorrected. The IR spectra were recorded using a JASCO FT/IR-410. The ${ }^{1} \mathrm{H}$ NMR (400 MHz) spectra, ${ }^{19} \mathrm{~F}$ NMR (376 MHz) spectra and ${ }^{13} \mathrm{C}$ NMR (100 $\mathrm{MHz}$ ) spectra were recorded in $\mathrm{CDCl}_{3}$ on a JEOL JNM-A400II FT NMR and the chemical shift, $\delta$, are referred to TMS $\left({ }^{1} \mathrm{H},{ }^{13} \mathrm{C}\right)$ and $\mathrm{CFCl}_{3}\left({ }^{19} \mathrm{~F}\right)$, respectively. The ${ }^{1} \mathrm{H}$ NMR (270 MHz) spectra and ${ }^{13} \mathrm{C}$ NMR (68 MHz) spectra were recorded on a JEOL JNM-A270II FT NMR. The EI-high-resolution mass spectra were measured on a JEOL JMS-700TZ. Optical rotation was measured with a Horiba High Sensitive Polarimeter. cis-1,2-Cyclohexanediol, 1,2-dodecanediol, $\quad(R, R)$-2,3-butanediol, 1,3-propanediol, 2,2-dimethyl-1,3-propanediol, $\quad N$-phenyl-2-ethanolamine $N, N$-diethylbenzamide, $\quad N, N$-diethyl-4-methyoxybenzamide, $N, N$-diethyl-3-methylbenzamide, and $N, N$-diethyl-4-chlorobenzamide were purchased from Tokyo Chemical Industry Co., Ltd. cis-1,2-Cyclododecanediol was separated by column chromatography from a mixture of the stereoisomers obtained from Tokyo Chemical Industry Co., Ltd. $\quad(R, R)$-Hydrobenzoin (99\%ee) was obtained from Aldrich. $\mathrm{Et}_{3} \mathrm{~N}-3 \mathrm{HF}$ was prepared according to the literature [8]. Dichlorotetrabutyldistannoxane was prepared according to the literature [9]. A 30 ml-Teflon FEP centrifuge tube with screw cup was obtained from Flon Industry and used as a reaction vessel in fluorination reaction.

\subsection{Preparation of $N, N$-diethyl-4-methoxybenzamide diethyl acetal}

To a $300 \mathrm{ml}$ three-necked glass flask equipped with mechanical stirrer, a reflux 
condenser, and a dropping funnel were introduced $N, N$-diethyl-4-methoxybenzamide (20.73 g, $100 \mathrm{mmol}$ ) and $\mathrm{CH}_{2} \mathrm{Cl}_{2}(50 \mathrm{ml})$ under $\mathrm{N}_{2}$ atmosphere. After addition of oxalyl chloride (12.95 g, $102 \mathrm{mmol}$ ) from the dropping funnel at room temperature, the reaction mixture was stirred under reflux for $90 \mathrm{~min}$. The mixture was cooled to -40 ${ }^{\circ} \mathrm{C}$, and a dry ethanol (27.64 g, $\left.600 \mathrm{mmol}\right)$ and $\mathrm{Et}_{3} \mathrm{~N}$ (20.60 g, $204 \mathrm{mmol}$ ) were added successively. Then, the temperature was allowed to rise up to room temperature and $\mathrm{CH}_{2} \mathrm{Cl}_{2}$ was carefully removed under reduced pressure. After the addition of hexane (100 ml), the precipitate was removed by filtration under $\mathrm{N}_{2}$ atmosphere. The filtrate was concentrated under reduced pressure and the residue was distilled to give $N, N$-diethyl-4-methoxybenzamide diethyl acetal (14.08 g, $50 \mathrm{mmol})$ in $50 \%$ yield (bp $94{ }^{\circ} \mathrm{C} / 0.42 \mathrm{mmHg}$ ). As it is moisture sensitive, it was kept and used as $1 \mathrm{M} \mathrm{CH}_{2} \mathrm{Cl}_{2}$ solution under $\mathrm{N}_{2}$. IR: (neat) $v$ 2972, 1609, 1509, 1244, $1083 \mathrm{~cm}^{-1}$. ${ }^{1} \mathrm{H}$ NMR (270 MHz) $\delta 0.97(\mathrm{t}, J=7.1 \mathrm{~Hz}, 6 \mathrm{H}), 1.17$ (t, $J=7.1 \mathrm{~Hz}, 6 \mathrm{H}), 2.60$ (q, $J=7.1 \mathrm{~Hz}, 4 \mathrm{H}), 3.25$ - 3.37 (m, 2H), 3.45 - 3.56 (m, 2H), 3.80 (s, 3H), 6.83 (d, $J=8.8 \mathrm{~Hz}, 2 \mathrm{H}), 7.41$ (d, $J=$ $8.8 \mathrm{~Hz}, 2 \mathrm{H}) .{ }^{13} \mathrm{C}$ NMR $(68 \mathrm{MHz}) \delta 15.05$ (2C), 16.05 (2C), 42.37 (2C), 55.14, 56.37 (2C), 109.02, 112.59 (2C), 128.37 (2C), 132.71, 158.67. HRMS (EI): calc. for $\mathrm{C}_{16} \mathrm{H}_{27} \mathrm{NO}_{3}$ : 281.1991 found: 281.1990 .

\subsection{1. $N, N$-Diethyl 4-methoxybenzamide ethylene acetal (1d)}

bp $85{ }^{\circ} \mathrm{C} / 0.28 \mathrm{mmHg}$. IR (neat): 2968, 1509, $1246 \mathrm{~cm}^{-1} . \quad{ }^{1} \mathrm{H}$ NMR (270 MHz) $\delta 0.98(\mathrm{t}, J=7.16,6 \mathrm{H}), 2.71(\mathrm{q}, J=7.11,4 \mathrm{H}), 3.73-3.78(\mathrm{~m}, 2 \mathrm{H}), 3.82(\mathrm{~s}, 3 \mathrm{H}), 4.01-$ 4.06 (m, 2H), $6.84-6.89$ (m, 2H), $7.45-7.48$ (m, 2H). ${ }^{13} \mathrm{C}$ NMR $\delta 14.18$ (2C), 40.34 (2C), 55.20, 63.85 (2C), 113.07 (2C), 119.75, 128.31 (2C), 133.13, 159.38. HRMS (EI): calc. for $\mathrm{C}_{14} \mathrm{H}_{20} \mathrm{NO}_{3}\left(\mathrm{M}^{+}-\mathrm{H}\right): 250.1443$ found: 250.1447 .

\subsection{Fluorination of diols}




\subsubsection{2-Fluoroethyl 4-methoxybenzoate (2d)}

A mixture of ethylene glycol $(62 \mathrm{mg}, 1 \mathrm{mmol})$ and a $\mathrm{CH}_{2} \mathrm{Cl}_{2}$ solution of $N, N$-diethyl-4-methoxybenzamide diethyl acetal (1.2 $\mathrm{ml}$ of $1 \mathrm{M}$ solution, $1.2 \mathrm{mmol})$ in a $30 \mathrm{ml}$-Teflon FEP tube was stirred at $50{ }^{\circ} \mathrm{C}$ under reduced pressure for $1 \mathrm{~h}$ to remove the generate ethanol and complete the transacetalization. To the crude 1d, $\mathrm{Et}_{3} \mathrm{~N}-3 \mathrm{HF}$ (322 mg, $2 \mathrm{mmol}$ ) was added and the mixture was stirred at $140{ }^{\circ} \mathrm{C}$ for $30 \mathrm{~min}$. The reaction mixture was cooled to room temperature and aq $\mathrm{NaHCO}_{3}(6 \mathrm{ml})$ was added. The product was extracted with ether, dried over $\mathrm{MgSO}_{4}$, and concentrated under reduced pressure. Purification by column chromatography (silica gel/hexane-ether) gave 2d (158 mg, $0.8 \mathrm{mmol}$ ) in 80 \% yield. IR: (neat) $v$ 2959, 1715, 1607, 1511, 1258, $1169 \mathrm{~cm}^{-1} .{ }^{1} \mathrm{H}$ NMR (400 MHz) $\delta 3.87(\mathrm{~s}, 3 \mathrm{H}), 4.59(\mathrm{dt}, J=28.8, J=4.2 \mathrm{~Hz}, 2 \mathrm{H})$, 4.68 (dt, $J=47.4, J=4.2 \mathrm{~Hz}, 2 \mathrm{H}), 6.93$ (d, $J=8.8 \mathrm{~Hz}, 2 \mathrm{H}), 8.04$ (d, $J=8.8 \mathrm{~Hz}, 2 \mathrm{H})$. ${ }^{13} \mathrm{C}$ NMR (100 MHz) $\delta$ 55.42, 63.54 (d, $\left.J=19.9 \mathrm{~Hz}\right), 81.55$ (d, $\left.J=170.4 \mathrm{~Hz}\right), 113.64$ (2C), 122.03 (2C), 131.79, 163.55, 166.08. ${ }^{19} \mathrm{~F}$ NMR (376 MHz) $\delta-225.01$ (tt, $J=$ 47.6, $J=28.7 \mathrm{~Hz}, 1 \mathrm{~F})$. $\quad$ HRMS (EI): calc. for $\mathrm{C}_{10} \mathrm{H}_{11} \mathrm{FO}_{3}$ : 198.0686 found 198.0692.

\subsubsection{2-Fluoroethyl 3-methylbenzoate (2a)}

IR: (neat) v 2958, 1725, 1596, $1276 \mathrm{~cm}^{-1} . \quad{ }^{1} \mathrm{H}$ NMR (400 MHz) $\delta 2.40(\mathrm{~s}, 3 \mathrm{H})$, $4.52-4.80$ (m, 4H) $7.31-7.38$ (m, 2H), 7.87 (d, $J=7.8 \mathrm{~Hz}, 1 \mathrm{H}), 7.88$ (s, $1 \mathrm{H}) .{ }^{13} \mathrm{C}$ NMR (100 MHz) $\delta 21.12,63.70(\mathrm{~d}, J=20.7 \mathrm{~Hz}), 81.39$ (d, $J=171.0 \mathrm{~Hz}), 126.80$, 128.23, 129.50, 130.15, 133.91, 138.14, 166.43. ${ }^{19} \mathrm{~F}$ NMR (376 MHz) $\delta-225.03$ (tt, $J$ $=47.6, J=28.7 \mathrm{~Hz}, 1 \mathrm{~F}) . \quad$ HRMS (EI): calc. for $\mathrm{C}_{10} \mathrm{H}_{11} \mathrm{FO}_{2}: 182.0743$ found 182.0743 .

\subsubsection{2-Fluoroethyl benzoate (2b)}

IR: (neat) $v 2958,1724,1276 \mathrm{~cm}^{-1} . \quad{ }^{1} \mathrm{H}$ NMR $\delta 4.53-4.81(\mathrm{~m}, 4 \mathrm{H}), 7.26-7.48$ (m, 2H), $7.56-7.60(\mathrm{~m}, 1 \mathrm{H}), 8.07-8.10(\mathrm{~m}, 2 \mathrm{H}) . \quad{ }^{13} \mathrm{C}$ NMR $(100 \mathrm{MHz}) \delta 63.83(\mathrm{~d}, J$ = $19.9 \mathrm{~Hz}$ ), 81.43 (d, $J=170.9 \mathrm{~Hz}), 128.42$ (2C), 129.66, 129.75 (2C), 133.24, 166.37. 
${ }^{19} \mathrm{~F}$ NMR (376 MHz) $\delta-225.09$ (tt, $\left.J=47.6, J=28.6 \mathrm{~Hz}, 1 \mathrm{~F}\right) . \quad$ HRMS (EI): calc. for $\mathrm{C}_{9} \mathrm{H}_{9} \mathrm{FO}_{2}: 168.0586$ found 168.0589 .

\subsubsection{2-Fluoroethyl 4-chlorobenzoate (2c)}

IR: (neat) v 2958, 1725, 1596, $1276 \mathrm{~cm}^{-1} . \quad{ }^{1} \mathrm{H}$ NMR (400 MHz) $\delta 4.52-4.80$ (m, 4H), $7.42-7.45(\mathrm{~m}, 2 \mathrm{H}), 8.00-8.03(\mathrm{~m}, 2 \mathrm{H}) .{ }^{13} \mathrm{C}$ NMR $(100 \mathrm{MHz}) \delta 64.03(\mathrm{~d}, J=$ $19.8 \mathrm{~Hz}), 81.31$ (d, $J=170.9 \mathrm{~Hz}), 128.06,128.78$ (2C), 131.13 (2C), 139.72, 165.49. ${ }^{19} \mathrm{~F}$ NMR $(376 \mathrm{MHz}) \delta-225.13(\mathrm{tt}, J=47.6, J=28.7 \mathrm{~Hz}, 1 \mathrm{~F})$. HRMS (EI): calc. for $\mathrm{C}_{9} \mathrm{H}_{8} \mathrm{ClFO}_{2}$ : 202.0197 found 202.0181.

\subsection{5. trans-2-Fluorocyclohexyl 4-methoxybenzoate (3)}

IR: (neat) v 2994, 1713, 1607, 1259, $1103 \mathrm{~cm}^{-1} . \quad{ }^{1} \mathrm{H}$ NMR (400 MHz) $\delta 1.34-$ 1.81 (m, 6H), 2.12 - 2.20 (m, 2H), 3.86 (s, 3H), 4.59 (dm, $J=50.3 \mathrm{~Hz}, 1 \mathrm{H}), 5.05-5.13$ (m, 1H), $6.91-6.94$ (m, 2H), $8.00-8.03(\mathrm{~m}, 2 \mathrm{H}) . \quad{ }^{13} \mathrm{C}$ NMR $(100 \mathrm{MHz}) \delta 22.76(\mathrm{~d}, J$ = $9.5 \mathrm{~Hz}$ ), 22.99, 29.38 (d, $J=6.1 \mathrm{~Hz}), 30.40$ (d, $J=18.3 \mathrm{~Hz}), 55.43,74.03$ (d, $J=19.5$ Hz), 91.93 (d, $J=178.5 \mathrm{~Hz}$ ), 113.55 (2C), 122.73, 131.70 (2C), 163.37, 165.62. ${ }^{19} \mathrm{~F}$ NMR (376 MHz) $\delta-182.11$ (brd, $J=45.2 \mathrm{~Hz}, 1 \mathrm{~F}$ ). $\quad$ HRMS (EI): calc. for $\mathrm{C}_{14} \mathrm{H}_{17} \mathrm{FO}_{3}$ : 252.1162 found 252.1162.

\subsubsection{1-Fluoro-2-dodecyl 4-methoxybenzoate (4a)}

IR: (neat) v 2926, 1714, 1607, $1257 \mathrm{~cm}^{-1} . \quad{ }^{1} \mathrm{H}$ NMR $(400 \mathrm{MHz}) \delta 0.87(\mathrm{t}, J=6.9$ Hz, 3H), $1.24-1.43$ (m, 16H), $1.67-1.83$ (m, 2H), 3.36 (s, 3H), 4.56 (dm, J = 45.0 Hz, 2H), $5.26(\mathrm{dm}, J=19.2 \mathrm{~Hz}, 1 \mathrm{H}), 6.93$ (d, $J=9.0 \mathrm{~Hz}, 2 \mathrm{H}), 8.02$ (d, $J=8.9 \mathrm{~Hz}, 2 \mathrm{H})$. ${ }^{13} \mathrm{C}$ NMR (100 MHz) $\delta 14.10,22.66,25.19,29.29,29.40$ (2C), 29.50, 29.55, 29.71 (d, $J$ = 5.5 Hz), 31.87, 55.43, 72.56 (d, $J=19.2 \mathrm{~Hz}), 83.77$ (d, $J=173.9 \mathrm{~Hz}), 113.59$ (2C), 122.47, 131.74 (2C), 163.45, 165.83. ${ }^{19} \mathrm{~F}$ NMR (376 MHz) $\delta-231.25$ (dt, $J=22.6, J=$ 47.7 Hz, 1F). $\quad$ HRMS (EI): calc. for $\mathrm{C}_{20} \mathrm{H}_{31} \mathrm{FO}_{3}$ : 338.2257 found: 338.2256. 


\subsubsection{2-Fluoro-1-dodecyl 4-methoxybenzoate (4b)}

White solid. $\quad \operatorname{mp~} 36{ }^{\circ} \mathrm{C}$. IR: $(\mathrm{KBr}) \vee 2926,1718,1607,1257 \mathrm{~cm}^{-1} .{ }^{1} \mathrm{H}$ NMR (400 MHz) $\delta 0.88(\mathrm{t}, J=6.8 \mathrm{~Hz}, 3 \mathrm{H}), 1.26-1.84(\mathrm{~m}, 18 \mathrm{H}), 3.87$ (s, 3H), $4.29-4.50$ (m, 2H), $4.79(\mathrm{dm}, J=49.0 \mathrm{~Hz}, 1 \mathrm{H}), 6.93$ (d, $J=9.0 \mathrm{~Hz}, 2 \mathrm{H}), 8.03$ (d, $J=8.8 \mathrm{~Hz}, 2 \mathrm{H})$.

${ }^{13} \mathrm{C}$ NMR (100 MHz) $\delta$ 14.10, 22.67, 24.81 (d, $\left.J=4.3 \mathrm{~Hz}\right), 29.31,29.35,29.42,29.51$, 29.57, 31.46 (d, $J=20.6 \mathrm{~Hz}), 31.88,55.43,66.01$ (d, $J=22.3 \mathrm{~Hz}), 91.58$ (d, $J=172.1$ $\mathrm{Hz}), 113.64$ (2C), 122.16, 131.77 (2C), 163.52, 166.06. ${ }^{19} \mathrm{~F}$ NMR (376 MHz) $\delta$ -187.52 - -187.13 (m, 1F). HRMS (EI): calc. for $\mathrm{C}_{20} \mathrm{H}_{31} \mathrm{FO}_{3}$ : 338.2257 found: 338.2262.

\subsection{8. trans-2-Fluorocyclododecanol (5)}

A $\mathrm{CH}_{2} \mathrm{Cl}_{2}$ solution of $N$, $N$-diethyl-4-methoxybenzamide diethyl acetal (1.2 ml of $1 \mathrm{M}$ solution, $1.2 \mathrm{mmol}$ ) and cis-1,2-cyclododecanediol (200 mg, $1 \mathrm{mmol}$ ) were introduced into a 30 ml-Teflon FEP tube, and a volatile part was removed under reduced pressure. Then $1 \mathrm{ml}$ of 1,2-dichloroethane was added and the reaction mixture was stirred at $50{ }^{\circ} \mathrm{C}$ for $1 \mathrm{~h}$ under reduced pressure. To the reaction mixture, $\mathrm{Et}_{3} \mathrm{~N}-3 \mathrm{HF}$ (325 mg, $2.1 \mathrm{mmol}$ ) was added and the mixture was stirred at $140{ }^{\circ} \mathrm{C}$ for 30 min. The reaction mixture was cooled to room temperature and aq $\mathrm{NaHCO}_{3}(6 \mathrm{ml})$ was added. The product was extracted with ether, dried over $\mathrm{MgSO}_{4}$, and concentrated under reduced pressure. The crude product, butanol (10 ml), and dichlorotetrabutyldistannoxane $(0.534 \mathrm{mg}, 1 \mathrm{mmol})$, and toluene $(5 \mathrm{ml})$ were introduced into a 25-ml glass flask equipped with a reflux condenser and the mixture was stirred under reflux. The transesterification reaction was monitored by GC and the disappearance of the 2-fluorocyclododecyl 4-methoxybenzoate was confirmed after 160 h. After removal of the volatile part under reduced pressure, 2-fluorocyclododecanol (112 mg, $0.56 \mathrm{mmol}$ ) was isolated by column chromatography (silica gel/hexane-ether) 
in $56 \%$ overall yield from the diol. White solid. mp $65{ }^{\circ} \mathrm{C}$. IR: (KBr) $\vee 3366$, 2933, 1470, $1022 \mathrm{~cm}^{-1} . \quad{ }^{1} \mathrm{H}$ NMR (400 MHz) $\delta 1.26-1.95(\mathrm{~m}, 20 \mathrm{H}), 2.21(\mathrm{t}, J=3.3$ $\mathrm{Hz}, 1 \mathrm{H}), 3.84-3.96(\mathrm{~m}, 1 \mathrm{H}), 4.56(\mathrm{dm}, J=49.5 \mathrm{~Hz}, 1 \mathrm{H}) .{ }^{13} \mathrm{C}$ NMR $(100 \mathrm{MHz}) \delta$ 20.35 (d, $J=3.3 \mathrm{~Hz}$ ), 20.57, 22.55, 22.59, 23.72, 23.76, 23.98, 24.01, 27.83 (d, $J=21.7$ $\mathrm{Hz}$ ), 28.73 (d, $J=5.6 \mathrm{~Hz}), 70.44$ (d, $J=18.4 \mathrm{~Hz}), 95.31$ (d, $J=166.1 \mathrm{~Hz}) .{ }^{19} \mathrm{~F}$ NMR (376 MHz) $\delta$-194.02 - -194.32 (m, 1F). HRMS (EI): calc. for $\mathrm{C}_{12} \mathrm{H}_{23} \mathrm{FO}$ : 202.1733 found: 202.1726 .

\subsection{9. (2R, 3S)-3-Fluoro-2-butyl 4-methoxybenzoate (6)}

IR: (neat) v 2988, 1713, 1258, 1168, $1101 \mathrm{~cm}^{-1} \cdot{ }^{1} \mathrm{H}$ NMR (400 MHz) $\delta 1.35-$ 1.44 (m, 6H), 3.86 (s, 3H), 4.78 (dm, $J=48.3 \mathrm{~Hz}, 1 \mathrm{H}), 5.16(\mathrm{dm}, J=18.3 \mathrm{~Hz}, 1 \mathrm{H}), 6.92$ $(\mathrm{d}, J=9.0 \mathrm{~Hz}, 2 \mathrm{H}), 8.01$ (d, $J=9.1 \mathrm{~Hz}, 2 \mathrm{H}) .{ }^{13} \mathrm{C}$ NMR $(100 \mathrm{MHz}) \delta 14.14(\mathrm{~d}, J=6.3$ Hz), 16.40 (d, $J=22.8 \mathrm{~Hz}), 55.24,71.94$ (d, $J=22.4 \mathrm{~Hz}), 90.83$ (d, $J=172.3 \mathrm{~Hz}$ ),

113.49 (2C), 122.50, 131.55 (2C), 163.37, 165.37. ${ }^{19}$ F NMR (376 MHz) $\delta-224.81-$ -225.21 (m, 1F). (2R, 3R)-isomer, -186.64 - -186.03 (m, 1F)). HRMS (EI): calc. for $\mathrm{C}_{12} \mathrm{H}_{15} \mathrm{FO}_{3}: 226.1005$, found 226.1008. $[\alpha]_{\mathrm{D}}^{22.4}=-10.0(\mathrm{c}=1.00, \mathrm{MeOH})$.

\subsubsection{0. (1R, 2S)-2-Fluoro-1,2-diphenylethyl 4-methoxybenzoate (7)}

White solid. $\quad$ mp $100{ }^{\circ} \mathrm{C}$. IR: (KBr) v 1709, 1258, $706 \mathrm{~cm}^{-1} .{ }^{1} \mathrm{H}$ NMR (400 MHz) $\delta 3.87$ (s, 3H), 5.85 (dd, $J=46.3, J=4.1 \mathrm{~Hz}, 1 \mathrm{H}$ ), 6.26 (dd, $J=18.1, J=4.2 \mathrm{~Hz}$, 1H), 6.93 (d, $J=8.9 \mathrm{~Hz}, 2 \mathrm{H}), 7.18-7.32(\mathrm{~m}, 10 \mathrm{H}), 8.02$ (d, $J=8.9 \mathrm{~Hz}, 2 \mathrm{H}) .{ }^{13} \mathrm{C}$ NMR (100 MHz) $\delta$ 55.36, 77.35 (d, $J=25.4 \mathrm{~Hz}), 94.44$ (d, $J=81.1 \mathrm{~Hz}), 113.65$ (2C), 122.09, 126.37, 126.44, 127.75 (2C), 127.99 (2C), 128.04 (2C), 128.41, 128.61, 131.76 (2C), 134.97 (d, $J=2.9 \mathrm{~Hz}), 135.59$ (d, $J=20.6 \mathrm{~Hz}), 163.57,164.90 .{ }^{19} \mathrm{~F}$ NMR (376 MHz) $\delta-188.91$ (dd, $J=46.3, J=18.3 \mathrm{~Hz}, 1 \mathrm{~F}$ ). $\mathrm{HRMS}$ (EI): calc. for $\mathrm{C}_{22} \mathrm{H}_{19} \mathrm{FO}_{3}$ : 350.1318 found: 350.1316. $[\alpha]_{\mathrm{D}}^{20.4}=+96.0(\mathrm{c}=1.00, \mathrm{MeOH})$. 
(1R, 2S)-2-Fluoro-1,2-diphenylethanol

Butanol (13 ml), 7 (298 mg, 0.85 mmol), dichlorotetrabutyldistannoxane (578 mg, $1.1 \mathrm{mmol})$, and toluene (5 ml) were introduced into a $25-\mathrm{ml}$ glass flask equipped with a reflux condenser, and the mixture was stirred under reflux for $90 \mathrm{~h}$. After removal of the volatile part under reduced pressure, (1R, 2S)-2-fluoro-1,2-diphenylethanol (330 mg, $0.65 \mathrm{mmol}$ ) was isolated by column chromatography (silica gel/hexane-ether). White solid. $\quad$ mp $98{ }^{\circ} \mathrm{C}$ (lit.[10] $99{ }^{\circ} \mathrm{C}$ ). IR: (KBr) 3578, 3033, 2880, 1452, 1050, 965, 706 $\mathrm{cm}^{-1} . \quad{ }^{1} \mathrm{H}$ NMR (400 MHz) $\delta 2.10$ (d, $\left.J=3.9 \mathrm{~Hz}, 1 \mathrm{H}\right), 4.98-5.03$ (m, 1H), 5.52 (dd, $J$ $=45.8, J=5.56 \mathrm{~Hz}, 1 \mathrm{H}), 7.22-7.35(\mathrm{~m}, 10 \mathrm{H}) .{ }^{13} \mathrm{C}$ NMR $(100 \mathrm{MHz}) \delta 76.31(\mathrm{~d}, J=$ $27.2 \mathrm{~Hz}), 96.21(J=177.8 \mathrm{~Hz}), 126.78$ (d, $J=7.2 \mathrm{~Hz}), 126.98$ (2C), 127.00 (2C), 128.19 (2C), 128.25 (2C), 128.79 (d, $J=1.7 \mathrm{~Hz}), 135.95$ (d, $J=19.9 \mathrm{~Hz}), 138.82$ (d, $J$ $=3.1 \mathrm{~Hz}) . \quad{ }^{19} \mathrm{~F}$ NMR $(376 \mathrm{MHz}) \delta-183.83(\mathrm{dd}, J=45.8, J=12.2 \mathrm{~Hz}, 1 \mathrm{~F}) . \quad[\alpha]_{\mathrm{D}}{ }^{22.1}$ $=+19.7(\mathrm{c}=1.00, \mathrm{MeOH})$.

\section{(1R, 2S)-2-Fluoro-1,2-diphenylethyl 3-methylbenzoate}

To a $\mathrm{CH}_{2} \mathrm{Cl}_{2}$ solution (3 ml) of 2-fluoro-1,2-diphenylethanol (66 mg, $0.3 \mathrm{mmol}$ ) in a $30 \mathrm{ml}$-Teflon FEP tube was added DFMBA (70 $\mathrm{mg}, 0.33 \mathrm{mmol}$ ) at $0{ }^{\circ} \mathrm{C}$, and the mixture was stirred at room temperature for $1 \mathrm{~h}$. To the reaction mixture, $2 \mathrm{ml}$ of water was added and the separated aqueous layer was extracted with ether three times. Organic layer was dried over $\mathrm{MgSO}_{4}$ and concentrated under reduced pressure. The residue was purified by column chromatography (silica gel/hexane-ether) to give 2-fluoro-1,3-diphenylethyl 3-methylbenzoate (87 mg, $0.26 \mathrm{mmol})$. $[\alpha]_{\mathrm{D}}^{24}=+60.7$ (c $=$ 1.00, MeOH) $\left\{\operatorname{lit}[1][\alpha]^{21}{ }_{\mathrm{D}}=+61.3(\mathrm{c}=1.02, \mathrm{MeOH})\right\}$

\subsubsection{3-Fluoro-1-propyl 4-methoxybenzoate (8)}

IR: (neat) v 2970, 1710, 1607, 1511, 1257, $1103 \mathrm{~cm}^{-1} .{ }^{1} \mathrm{H}$ NMR (400 MHz) $\delta$ $2.16(\mathrm{dm}, J=25.7 \mathrm{~Hz}, 2 \mathrm{H}), 3.87(\mathrm{~s}, 3 \mathrm{H}), 4.43(\mathrm{t}, J=6.2 \mathrm{~Hz}, 2 \mathrm{H}), 4.62(\mathrm{dt}, J=46.9, J=$ 
$5.9 \mathrm{~Hz}, 2 \mathrm{H}), 6.92$ (d, $J=9.0 \mathrm{~Hz}, 2 \mathrm{H}), 7.99$ (d, $J=8.9 \mathrm{~Hz}, 2 \mathrm{H}) .{ }^{13} \mathrm{C}$ NMR $(100 \mathrm{MHz})$ $\delta 29.81$ (d, $J=19.8 \mathrm{~Hz})$, 55.26, 60.44 (d, $J=5.7 \mathrm{~Hz}), 80.61$ (d, $J=165.2 \mathrm{~Hz}), 113.50$ (2C), 122.37, 131.45 (2C), 163.31, 166.03. ${ }^{19}$ F NMR (376 MHz) $\delta-222.57$ (tt, $J=$ 47.0, $J=25.7 \mathrm{~Hz}, 1 \mathrm{~F}) . \quad$ HRMS (EI): calc. for $\mathrm{C}_{11} \mathrm{H}_{13} \mathrm{FO}_{3}$ : 212.0849 found: 212.0841 .

\subsubsection{3-Fluoro-2,2-dimethylpropyl 4-methoxybenzoate (9)}

IR: (neat) $v$ 2968, 1714, 1607, $1258 \mathrm{~cm}^{-1} .{ }^{1} \mathrm{H}$ NMR (400 MHz) $\delta 1.05$ (d, $J=$ $1.8 \mathrm{~Hz}, 6 \mathrm{H}), 3.84$ (s, 3H), 4.14 (d, $J=1.1 \mathrm{~Hz}, 2 \mathrm{H}), 4.27$ (d, $J=47.9 \mathrm{~Hz}, 2 \mathrm{H}), 6.92$ (d, $J$ $=9.0 \mathrm{~Hz}, 2 \mathrm{H}), 7.99(\mathrm{~d}, J=8.9 \mathrm{~Hz}, 2 \mathrm{H}) .{ }^{13} \mathrm{C} \mathrm{NMR}(100 \mathrm{MHz}) \delta 20.63(\mathrm{~d}, J=5.0 \mathrm{~Hz}$, 2C), 35.87 (d, $J=17.4 \mathrm{~Hz}$ ), 55.26, 68.56 (d, $J=4.1 \mathrm{~Hz}$ ), 88.10, 113.54 (2C), 122.44, 131.42 (2C), 163.32, 165.92. ${ }^{19} \mathrm{~F}$ NMR (376 MHz) $\delta-226.89(\mathrm{t}, J=47.6 \mathrm{~Hz}, 1 \mathrm{~F})$. HRMS (EI): calc. for $\mathrm{C}_{13} \mathrm{H}_{17} \mathrm{FO}_{3}$ : 240.1162 found: 240.1160 .

\subsubsection{N-(2-Fluoroethyl)-N-phenyl 4-methoxybenzamide (10)}

IR: (neat) v 2959, 2839, 1642, 1606, $1254 \mathrm{~cm}^{-1} . \quad{ }^{1} \mathrm{H}$ NMR (400 MHz) $\delta 3.74$ (s, 3H), 4.19 (dt, $J=25.6, J=4.9 \mathrm{~Hz}, 2 \mathrm{H}), 4.75$ (dt, $J=47.5, J=4.9 \mathrm{~Hz}, 2 \mathrm{H}), 6.66$ (d, $J=$ $8.9 \mathrm{~Hz}, 2 \mathrm{H}), 7.09-7.29(\mathrm{~m}, 7 \mathrm{H}) .{ }^{13} \mathrm{C} \mathrm{NMR}(100 \mathrm{MHz}) \delta 51.58(\mathrm{~d}, J=21.4 \mathrm{~Hz})$, 55.10, 81.43 (d, $J=169.0 \mathrm{~Hz}$ ), 112.91 (2C), 126.48, 127.47 (2C), 127.58, 129.14 (2C), 130.90 (2C), 144.46, 160.67, 170.12. ${ }^{19}$ F NMR (376 MHz) $\delta-222.81--222.43$ (m, 1F). HRMS (EI): calc. for $\mathrm{C}_{16} \mathrm{H}_{16} \mathrm{FNO}_{2}$ : 273.1165 found: 273.1159.

\subsubsection{N-(2-Fluorooctyl)-N-phenyl 4-methoxybenzamide (11)}

White solid. $\quad \operatorname{mp~} 47^{\circ} \mathrm{C}$. IR: (KBr) $v 2931,2857,1644,1606,1254 \mathrm{~cm}^{-1} \cdot{ }^{1} \mathrm{H}$ NMR (400 MHz) $\delta 0.88$ (t, $J=6.8 \mathrm{~Hz}, 3 \mathrm{H}), 1.29-1.72$ (m, 10H), 3.70 (s, 3H), $3.71-$ $3.78(\mathrm{~m}, 1 \mathrm{H}), 4.30$ (ddd, $J=33.7, J=14.4, J=2.1 \mathrm{~Hz}, 1 \mathrm{H}), 4.95(\mathrm{dm}, J=51.0 \mathrm{~Hz}, 1 \mathrm{H})$, $6.64(\mathrm{~d}, J=8.9 \mathrm{~Hz}, 2 \mathrm{H}), 7.09-7.29(\mathrm{~m}, 7 \mathrm{H}) .{ }^{13} \mathrm{C}$ NMR $(100 \mathrm{MHz}) \delta 13.93,22.40$, 24.69 (d, $J=3.0 \mathrm{~Hz}), 28.92,31.52,32.85$ (d, $J=19.9 \mathrm{~Hz}), 54.99,55.48$ (d, $J=21.4 \mathrm{~Hz}$ ), 
92.13 (d, $J=170.5 \mathrm{~Hz}), 112.83$ (2C), 126.26, 127.42 (2C), 127.72, 128.97 (2C), 130.82 (2C), 144.80, 160.56, 170.09. ${ }^{19} \mathrm{~F}$ NMR (376 MHz) $\delta-184.97$ - -184.58 (m, 1F). HRMS (EI): calc. for $\mathrm{C}_{22} \mathrm{H}_{28} \mathrm{NO}_{2} \mathrm{~F}: 357.2104$ found: 357.2103 .

\section{References}

[1] A. Yoneda, T. Fukuhara, S. Hara, Chem. Commun. (2005) 3589-3590.

[2] R. A. McClelland, S. Gedge, J. Bohonek, J. Org. Chem. 46 (1982) 886-891.

[3] R. A. McClelland, M. Ahmad, J. Org. Chem. 44 (1979) 1855-1860.

[4] R. F. Abdulla, R. S. Brinkmeyer, Tetrahedron 35 (1979) 1675-1735.

[5] T. Inagaki, T. Fukuhara, S. Hara, Synthesis (2003) 1157-1159.

[6] J. Otera, N. Dan-oh, H. Nozaki, J. Org. Chem. 56 (1991) 5307-5311.

[7] T. Nomoto, T. Fukuhara, S. Hara, Synlett (2006) 1744-1746.

[8] S. Ayuba, N. Yoneda, T. Fukuhara, S. Hara, Bull. Chem. Soc. Jpn., 75 (2002) 1597-1603.

[9] D. L. Tierney, P. J. Moehs, D. L. Hasha, J. Organometal. Chem. 620 (2001) 211-226.

[10] S. Rozen, O. Lerman, J. Org. Chem. 45 (1980) 672-678. 